\title{
Silver diagnosis in neuropathology: principles, practice and revised interpretation
}

\author{
Toshiki Uchihara
}

Received: 24 December 2006 / Revised: 16 January 2007 / Accepted: 18 January 2007 / Published online: 31 March 2007 (C) Springer-Verlag 2007

\begin{abstract}
Silver-staining methods are helpful for histological identification of pathological deposits. In spite of some ambiguities regarding their mechanism and interpretation, they are widely used for histopathological diagnosis. In this review, four major silver-staining methods, modified Bielschowsky, Bodian, Gallyas (GAL) and CampbellSwitzer (CS) methods, are outlined with respect to their principles, basic protocols and interpretations, thereby providing neuropathologists, technicians and neuroscientists with a common basis for comparing findings and identifying the issues that still need to be clarified. Some consider "argyrophilia" to be a homogeneous phenomenon irrespective of the lesion and the method. Thus, they seek to explain the differences among the methods by pointing to their different sensitivities in detecting lesions (quantitative difference). Comparative studies, however, have demonstrated that argyrophilia is heterogeneous and dependent not only on the method but also on the lesion (qualitative difference). Each staining method has its own lesion-dependent specificity and, within this specificity, its own sensitivity. This "method- and lesion-dependent" nature of argyrophilia enables operational sorting of disease-specific lesions based on their silver-staining profiles, which may potentially represent some disease-specific aspects. Furthermore, comparisons between immunohistochemical and biochemical data have revealed an empirical correlation between GAL+/CS-deposits and 4-repeat (4R) tau (corticobasal degeneration, progressive supranuclear palsy and
\end{abstract}

T. Uchihara $(\bowtie)$

Department of Neuropathology,

Tokyo Metropolitan Institute for Neuroscience,

2-6 Musashi-dai, Fuchu, Tokyo 183-8526, Japan

e-mail: uchihara@tmin.ac.jp argyrophilic grains) and its complementary reversal between GAL-/CS+deposits and 3-repeat (3R) tau (Pick bodies). Deposits containing both $3 \mathrm{R}$ and $4 \mathrm{R}$ tau (neurofibrillary tangles of Alzheimer type) are GAL+/CS+. Although no molecular explanations, other than these empiric correlations, are currently available, these distinctive features, especially when combined with immunohistochemistry, are useful because silver-staining methods and immunoreactions are complementary to each other.

Keywords Argyrophilia - Gallyas - Campbell-Switzer · Tauopathies $\cdot$ Synucleinopathies $\cdot$ Senile plaques ·

Methenamine

\section{Introduction}

The year 2006 was the centenary anniversary of the first clinical description of a presenile patient with progressive cognitive decline, later defined as Alzheimer's disease (AD) [7]. It was the same year (1906) when Santiago Ramón y Cajal and Camillo Golgi were awarded the Nobel prize for their contributions on neuroanatomy achieved by using silver-staining methods, especially Golgi-silver technique. In the field of neuropathology as well, silver staining was the principal method of choice at that time, which visualized senile plaques (SPs), neurofibrillary tangles (NFTs) and Pick bodies [2]. More than 80 years have passed before biochemical compositions of these pathological structures were unveiled. During this period, technical trials to identify and localize these molecules on histological sections showed the effectiveness of immunohistochemistry and revolutionized not only research but also routine diagnosis in the field of neuropathology. Probably because of the apparent success in the molecular explanation of diseases, 
recent attention to silver staining has not been very enthusiastic. Although most silver-staining methods are still left with some ambiguities, neuropathologists have profited by their efficacies. Silver-staining methods have been steadily improved and one of the recent advances is undoubtedly Gallyas method [41]. In practice, it allows stable, reproducible and sensitive visualization with low background. However, it is unfortunate that their principles, technical details and interpretations, which are different according to each method, are sometimes not sufficiently shared among neuropathologists and technicians. It frequently happens that some time-honored protocols are left without possible explanations and their interpretation is often restricted to "argyrophilic or not". This naive interpretation is based on the assumption that "argyrophilia" is a homogeneous phenomenon irrespective of the staining method and the lesion. Recent studies have demonstrated, on the contrary, that "argyrophila" is heterogeneous and dependent on the staining method and the lesion [85, 119-122]. Here, established silver-staining methods in the field of neuropathology are reconsidered with the intension of improving their use and interpretation.

The first part of this review summarizes basic principles and outlines protocols about four principal silver-staining methods for formalin-fixed, paraffin-embedded samples from human autopsy brains mainly with degenerative diseases, currently in use in our laboratory and their modifications. Although other silver-staining methods have also been invented to visualize normal neuronal structures, astrocytes, microglia and experimental tissue samples, it is not possible to encompass the whole range of these techniques mainly because of the limitation of my own experience. The second part provides an overview of similarities and differences between silver-staining methods and immunohistochemistry, so that a comprehensive interpretation, including their pitfalls results. Nevertheless, this review is not intended to establish a relative grading of these methods by identifying the possible superiority of a method (for example, immunohistochemistry) over another (for example, a silver-staining method). Instead, it is designed to show how unique each staining method can be, by providing a distinct point of view, each independent of the other. If we are prepared to take these possible differences of staining methods into account, such an approach will provide a less biased, multi-faceted and fuller stereoscopic picture than can be achieved with immunohistochemistry alone.

\section{General principle of silver staining}

The coloration in conventional histology is based on the selective affinity of a dye to some tissue elements relative to surrounding elements and their permeability. This differential affinity to specific tissue elements is finally represented by the distinctive original color of the dye as seen with its solution. This is essentially a single step procedure, called dyeing, and does not involve chemical transformation of the dye. Subsequent washing step is also selective in dissolving out the attached dye, which is called differentiation [5]. Various combinations (for example, hematoxylin and eosin, the Klüver-Barrera method, etc.) are currently in use for neuropathological examinations. The mechanism of silver staining is, however, completely different. Because the solutions containing coloring agents used for silver-staining methods are usually colorless, simple application of these solutions to tissue sections, by itself, does not allow visualization through light microscope even if the reagent has some affinity to the tissue elements. Subsequent chemical transformation is necessary for visualization. In silver solutions, most of the silver is present as ion or complex salt. The silver ions should be first attached to the target elements as silver ions or silver salts (step 1). This attachment, however, does not allow visualization by itself. The attached silver ions $(\mathrm{Ag}+)$ have to be reduced (=accept electrons) to yield, in situ, metallic silver particles (Ag) now visualized only if their amount and size are sufficient for microscopic detection (step 2). Therefore, not only the differential affinity of the silver ion to some tissue elements (step 1) but also its chemical transformation for visualization (step 2) is essential. Although the attachment of silver ions to tissue elements is based on the "in situ" formation of silver salt, these silver ions, either in the solution or as salts, are reducible. They undergo reduction to form metallic silver. Gallyas demonstrated that there is a time lag between the rapid attachment of silver ions onto the section (step 1, within $15 \mathrm{~min}$ ) and delayed formation of metallic silver particles (step 2) there [42]. Because these two steps are independently affected by $\mathrm{pH}$, temperature, the concentration of silver ion and coexisting molecules [42], these steps are independent even if both take place in a single manipulation. This dual function of silver, that enables "site-directed chemical transformation", is fundamental to various silver-staining methods.

Because some tissue elements (glutathione, creatine, adrenaline etc.) exhibit an intrinsic reducing capacity, which may be strong enough to reduce silver ions into metallic silver particles in situ, these two steps (selective attachment and visualization after reduction of silver ions) may take place in a single manipulation. This is called silver reaction or "argentaffin" reaction [35, 102]. When the reducing capacity of the tissue elements is absent or not sufficient, it is necessary to provide reducing agents externally after silver ions have been attached to the tissue elements. It is also possible to facilitate or modify these steps by some reagents [40]. Anyway, this two-step procedure is 
called postblackening (Nachschwärzen) or "argentophilic" reaction [35, 102] and most of the current silver-staining methods for neuropathology consist, in essence, of at least these two steps. If sufficient amount of silver ions have been attached to the tissue elements but not have been reduced spontaneously after step 1 , it is possible to visualize them simply by applying an appropriate reducing agent externally. This is called chemical development, during which the amount of deposited silver in the tissue section remains unchanged. However, it is not easy to increase the amount of silver deposits during step 1. For example, increasing the concentration of silver reagents or prolongation of incubation time is frequently hampered by nonspecific staining. This trade off between sensitivity and specificity, principally encountered in all kinds of staining, is especially troublesome with silver-staining methods because reactions in the silver solutions and those in reducing agents are both capricious and difficult to standardize. This is one of the reasons why more sensitive, stable and reproducible silver solutions and staining methods have been a matter of considerable endeavor for a long period, as will be discussed later.

\section{Particle size and possible determinants of selective staining}

It has been reported that the apparent color of silver-stained elements is correlated with the size of final silver particles. In the test tube, the smallest range in their diameter (10$20 \mathrm{~nm}$ ) is correlated with yellow color. The largest range $(>100 \mathrm{~nm})$ is associated with black color. Intermediate ranges are correlated with red $(>30 \mathrm{~nm})$ or blue-purple $(50-$ $60 \mathrm{~nm}$ ) [102, 103], as reported by Liesegang [70]. The emission spectra of fluorogenic semiconductor nanocrystals, recently introduced for fluorolabeling, are dependent not only on their materials but also on their particle size $[20,22]$. Although the size range of semiconductor nanocrystals $(2-6 \mathrm{~nm})$ is much smaller than that of silver particles, it is interesting to note that these differences in their color spectra are dependent on the particle size in both silver particles and the nanocrystals. Others reported, however, that black color is correlated with a larger amount of deposits [35]. Although the deposition of silver particles is primed by an affinity of the silver ion to some tissue elements, is this affinity the only major determinant of the selective staining? According to Seki [102], some colloidal substance in the tissue may hinder formation and deposition of metallic silver particles (Schutzkolloid). In addition, packing density of tissue ultrastructures (for example, fiber density) may also be responsible, because an excessive looseness of the structure may not allow secure settlement of silver particles. It is also possible that its extreme tight- ness does not permit appropriate growth of silver particles. He then concluded that a small quantity of this intervening colloid (eine Armut an Schutzkolloid) and an appropriate density of the structure (eine passende Strukturdichte) of the target structure are also important for the selectivity of silver staining. This idea that some spatial constraints around silver particles, in addition to the primary affinity of the silver ion, are essential in determining the selectivity of silver staining may be related to the current concept of disease-specific "conformational status" of pathological deposits and their constituent molecules.

These explanations are, however, far from complete for the majority of silver-staining methods. Furthermore, because these experiments have been performed mainly in animal tissues, in which sampling and handling of tissue materials are optimal, it remains to be clarified whether similar explanations are applicable to human samples obtained for neuropathological examinations, often containing distinct pathological lesions and suffering from suboptimal handling such as postmortem delay, suboptimal fixation and embedding in paraffin wax. Even though various methods for celloidin- or paraffin-embedded autopsy samples have been invented. Table 1 shows fundamental steps and contents of the solutions for the silver-staining methods currently in use in our laboratory.

\section{Bielschowsky methods}

Improvements in silver-staining methods have often been derived from chemistry. The ammoniacal silver solution had been used for production of mirror, based on the nature of the complex "silver diamine $[\mathrm{Ag}(\mathrm{NH} 3) 2]^{+}$" in the solution that is liable to precipitate, after being reduced, at the interface between the glass surface and the solution. This is called the silver mirror reaction and it was Fajersztajn who initially applied this phenomenon to tissue staining [34]. Later, Max Bielschowsky [8] improved this method by incubating formalin-fixed frozen sections in the silver nitrate prior to the ammoniacal silver solution (Table 1). Although the exact mechanism still remains to be clarified [100], it is plausible that this prior treatment with the silver nitrate provides active foci for subsequent silver deposition in the ammoniacal silver solution [50]. Anyway, it is reasonable to suppose that this stepwise impregnation (silver nitrate-ammoniacal silver) improved the staining probably by increasing the amount and the size of silver precipitates [66]. Early modifications of Bielschowsky method were summarized by Beech and Davenport [6]. Garven and Gairns [50] provided a comprehensive review on the possible mechanism of the so-called Bielschowsky-Gros method and its modifications. The presence of a huge number of modifications, however, indicates that this method is highly 


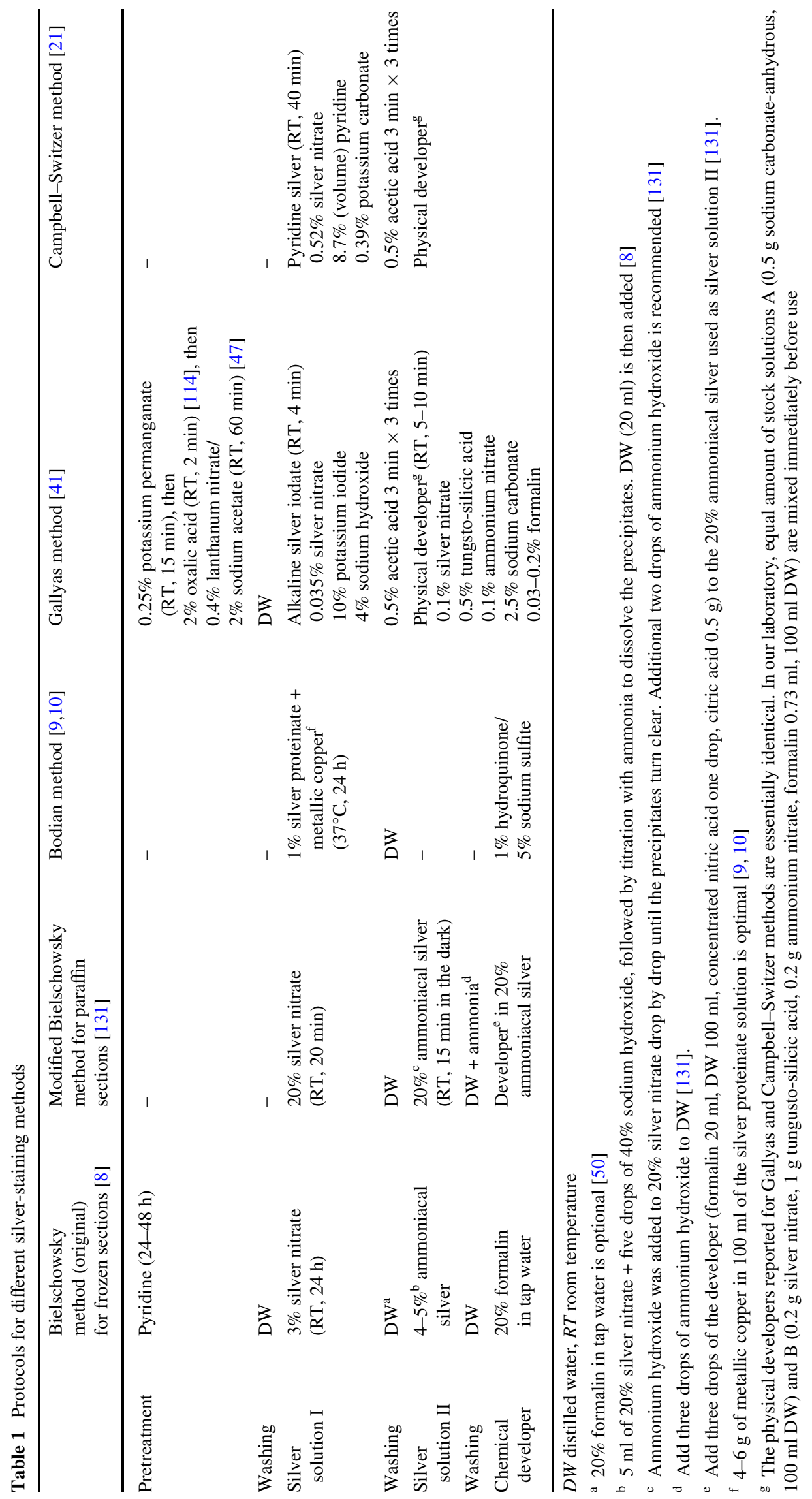


unstable and still awaits improvements. A modification for paraffin-embedded samples of normal neural tissues from various animal species was first proposed by Sevier and Munger [105]. Practically, it is probably YamamotoHirano's modification [131], which is currently accepted as the standard Bielschowsky method for neuropathological examination of paraffin-embedded autopsy samples (Table 1). This Yamamoto-Hirano's modification is different from other conventional modifications of Bielschowsky method because the usual silver nitrate-ammoniacal silver sequence is followed by another ammoniacal silver solution containing developers.

\section{Bodian method}

Bodian established a new method, applicable to paraffinembedded sections, for staining nerve fibers and nerve endings $[9,10]$. This method is rather stable and reproducible and is currently in use for neuropathology. He used $1 \%$ silver proteinate (which usually contains $8 \%$ of silver) solution and placed metallic copper in it. Because the staining does not work well in the absence of metallic copper, he speculated that metallic copper might regulate the deposition of metallic silver by decreasing the concentration of silver ion $[9,10]$. However, the mixture of silver nitrate and powdered egg albumin was reportedly successful even in the absence of metallic copper [92]. Nevertheless, the measured concentration of silver ion gradually decreases after addition of metallic copper, as demonstrated by two independent groups $[64,93]$. Concomitant and gradual increase in the concentration of copper ion $[64,93]$ is accompanied by a decrease in $\mathrm{pH}$ [93]. These reciprocal changes between concentrations of silver and copper ions are mainly explained by a primary difference in ionization energy between copper and silver. In other words, copper is more liable to be ionized than silver. Because their concentrations are not influenced by the presence of sections in the solution, the amount of silver ion trapped in the section is practically negligible. While the addition of metallic copper up to $5 \mathrm{~g} / 100 \mathrm{ml}$ progressively decreases the concentration of silver ion in dose-dependent fashion (from 1,000 to $250 \mu \mathrm{g} / \mathrm{ml}$ ), further increase in the amount of metallic copper up to $15 \mathrm{~g} / 100 \mathrm{ml}$ does not lead to further increase in the copper ion nor further decrease in the silver ion in the solution [64]. Indeed, this amount of copper, $5 \mathrm{~g} / 100 \mathrm{ml}$, is in perfect agreement of the amount, 4-6 g/100 ml, originally recommended by Bodian $[9,10]$ and the amount of metallic copper more or less than this range does not give optimal results. Interestingly, not only the amount of metallic copper but also the shape of metallic copper (foil, plate, chip or granules) influences the concentration of copper and silver ions and the results of staining. The metallic copper as "foil" is correlated with the highest concentration of copper ion and the most intense staining [64, 93]. Moreover, these reactions in the silver proteinate solution occur slowly and the equilibrium is reached after 12-16 h. Figure 1 illustrates putative chronological events during incubation in the silver proteinate solution and development. In the early phase of silver impregnation, the high concentration of silver ion facilitates its attachment to the section (step 1, Fig. 1). This initial attachment is apparently not selective, because early interruption of incubation yields an overall nonselective nerve staining [93]. Gradual deposition of silver ion as metallic silver on the surface of metallic copper (step 2, Fig. 1) leads to a gradual decrease in the concentration of silver ion and a concomitant increase in the concentration of copper ion in the solution. Continued incubation in the solution with a decreasing concentration of silver ion facilitates liberation of the silver ion, once trapped in the section, back into the solution (step 3, Fig. 1). The selective staining of Bodian method is explained if this rate of liberation of silver ion back to the solution (step 3, Fig. 1) is different according to the tissue elements. This chronological change may be necessary to yield the selective staining and explains why an equivalent concentration of copper ion provided as copper sulfate instead of metallic copper does not yield similar results [64]. Some copper ions are also deposited onto the section (step 4, Fig. 1). Residual silver proteinate as colloidal fraction may release silver ions gradually throughout the incubation (step 5, Fig. 1). For practice, (1) $5 \mathrm{~g}$ of metallic copper as foil in $100 \mathrm{ml}$ of $1 \%$ silver proteinate solution and (2) incubation for 16-24 h are recommended as optimal for Bodian method [64].

After being incubated in the silver proteinate solution, sections are treated with $1 \%$ hydroquinone with $5 \%$ sodium sulfite for reduction (step 6, Fig. 1) [9, 10]. It has been reported that formaldehyde, suitable for reducing the silver diamine of Bielschowsky method, is not appropriate by itself for reducing the silver ions in Bodian method. In contrast, hydroquinone suitable to reduce the silver ions in Bodian method is not appropriate for reducing the silver diamine of Bielschowsky method [35]. However, some recommended formaldehyde in combination with hydroquinone for the reducing step of Bodian method [9, 10]. Because it is usually after the incubation in the developer when the sections appear contrasted for visualization, it seems reasonable to suppose that deposited silver ions are reduced to form metallic silver only after exposure to hydroquinone. If this is the case, treatment of the silver impregnated sections with sodium sulfite, which dissolves out silver salt but not metallic silver, will diminish the staining. On the contrary, Peters [93] demonstrated the presence of sodium sulfite-resistant metallic silver and suggested that at least some fraction of silver ions have already been reduced to metallic silver during incubation in the 


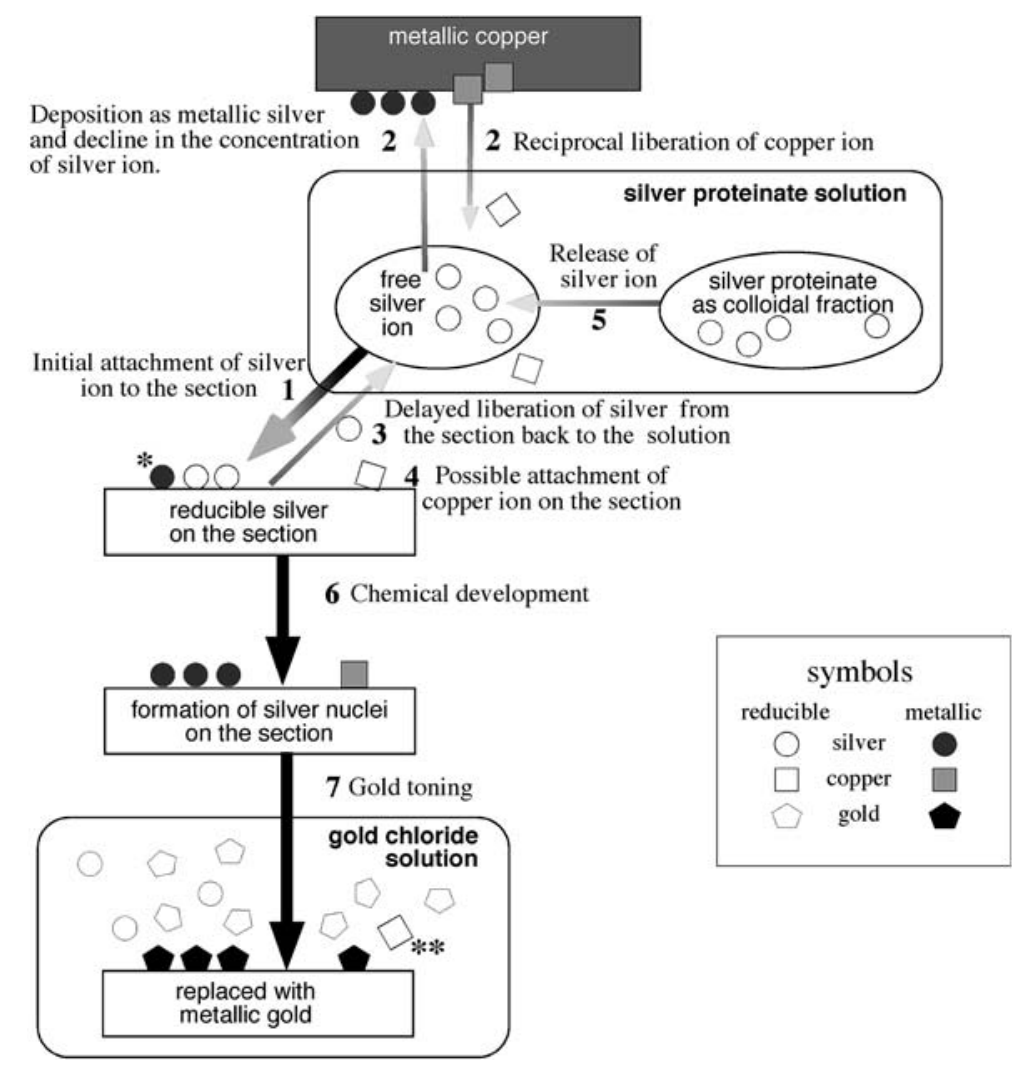

Fig. 1 Mechanism of the Bodian method. Step 1: Attachment of silver ions to the section driven by high concentration of silver ions; single asterisk: a fraction of silver ions are already reduced to metallic silver even before chemical development. Step 2: Deposition of silver ions as metallic silver leads to a decrease in the concentration of silver ion and reciprocal liberation of copper ions. Step 3: Delayed liberation of silver ions from the section back to the solution with decreased concentration of silver ion after step 2. Step 4: Copper ions are now available in the

silver proteinate solution (asterisk, step 4, Fig. 1). They may serve as nuclei that attract surrounding reducible silver ions/salts to form larger metallic silver particles during subsequent development. Indeed, Gallyas demonstrated that radiolabeled silver ions in the silver proteinate solution are deposited to the section in the form of metallic silver even before the development. This is based on the finding that the radiolabeled silver on the section is resistant to the treatment with $1 \%$ silver nitrate/10\% acetic acid that washes out reducible silver ions but not metallic silver particles. Because the localization of this radiolabeled silver, now deposited as metallic silver, is in good agreement with the final image of the adjacent section stained by Bodian method, it is likely that these metallic silver deposits play a determinant role in defining final image obtained with Bodian method [44]. At any rate, it is possible to intensify the staining through subsequent gold toning by incubating in $1 \%$ gold chloride with glacial acetic acid (three drops for $100 \mathrm{ml}$ ). Although the mechanism of Bodian method, espe- solution after step 2 to be attached on the section. Step 5: Silver proteinate gradually releases silver ions during steps $1-4$. Step 6: Not only silver but also copper ions/salts on the section are reduced to metallic particles. Step 7: These metallic particles are replaced with metallic gold; double asterisk: retrieval of copper ions in the gold chloride solution indicates that metallic copper has been deposited on the section (steps 4 and 6). Symbols: circle: silver; square: copper; pentagon: gold; blank symbols: reducible (ion/salts); filled symbols: metallic cially that of copper ion, is not yet completely clarified, not only silver but also copper ions are retrieved in the gold chloride solution for toning (double asterisk, step 7, Fig. 1) [64]. This indicates that the ionized copper in the silver proteinate solution has been deposited on the tissue and that the deposited copper, in addition to silver, is replaced with gold [64]. This dual replacement may explain why this gold toning is necessary and particularly effective for Bodian method. After gold toning, it is now possible and necessary to treat the sections with $5 \%$ sodium thiosulfate to dissolve out silver ions that remain unreduced in the tissue. Otherwise, these remaining ions may be reduced after exposure to light to form undesirable precipitates. Because Bodian method was originally invented to stain normal axons and neurofibrils, these normal structures are always superimposed on pathological structures such as SPs and NFTs. These intervening normal structures are one of the major hindrances of Bodian method especially when examining silver-stainable deposits overlaid on normal structures. 


\section{Gallyas method}

Another way of improvement had been achieved based on the chemical principle of photography, "physical development". As opposed to the chemical development, additional silver ions are provided with reducing agents to form silver particles around the reactive foci, which have been already activated after exposure to light in dose-dependent manner. This greatly increases the amount of metallic silver or oxidized silver that are now visible as photographic images. It was Liesegang who first introduced this idea to histological staining in an attempt to establish a more consistent and reproducible method of silver staining $[70,88]$. The protocols with physical development were later customized successfully for neuropathology by Gallyas [40]. Because physical developer solutions contain, in general, silver ions and reducing agents that together react rapidly, protecting colloid (tungsto-silicic acid for Gallyas method) is included in order to control by retarding this reaction [43].

As with other silver-staining methods, the attachment of silver ion to specific targets determines final localization of silver particles. According to Gallyas, this specific attachment is mediated by some determinant substance, which binds to the target tissue elements or the tissue elements themselves, followed by their chemical modifications. These foci are enabled to attract silver ions to form silver salts. Otherwise, the determinant substance renders these foci capable of reducing silver ions to form submicroscopic metallic silver particles bound to the target elements. The chemical nature of the original determinant substance may determine which components (for example, fibrous astrocytes, microglia or oligodendrocytes) in the section are finally silver-stained [37-39]. For visualization of NFTs, sections are incubated with silver iodide in alkaline condition that exhibits affinity to NFTs [41] (Table 1). Although Gallyas customized protocols according to various target structures other than NFTs [37-39], this silver iodide protocol for NFTs, currently in routine use for neuropathological diagnosis, is defined in this review as "Gallyas method" for simplicity and convenience. If this silver solution is prepared with radiolabeled silver, it is possible to quantify the silver ions/particles after each step of the staining protocol. Gallyas demonstrated that most of the silver attached to the section after incubation in this silver iodide solution is reducible silver salt but not metallic silver, because it is washed out after incubation in nonlabeled $1 \%$ silver nitrate/ $10 \%$ acetic acid (Table 1 of Ref. [44]), a treatment that expels reducible silver ions/salts from the sections. Incubation in sodium thiosulfate or sodium cyanide, alternate ways to expel these reducible silvers, diminishes the final image, again compatible with the assumption that the attached silver after incubation in the silver iodide solution is silver ions/salts but not metallic silver [41].
Is a separate step necessary to transform these attached silver ions/salts into metallic silver particles so that they may subsequently work as nuclei for subsequent growth of silver particles in the physical developer? Because the reducing agent in the physical developer is ready to reduce these attached silver ions/salts into metallic silver nuclei, an isolated step for reduction is not necessary before the treatment in the physical developer. However, a brief exposure of the silver-impregnated sections to formalin prior to the physical development leads to an instantaneous completion of the physical development [41]. However, it is possible that silver ions contained in the physical developer potentially attaches to the tissue and are physically developed to form separate metallic silver particles. Because this may happen independently of the silver from the initial alkaline silver iodide solution [45], a prolonged incubation in the physical developer may be hampered by this unexpected staining. Therefore, pretreatments have been invented in order to minimize this background. A pretreatment initially proposed by Gallyas was 5\% periodic acid for $30 \mathrm{~min}$. He tried various pretreatments [46] and finally proposed an alternative pretreatment with $0.4 \%$ lanthanum nitrate $/ 2 \%$ sodium acetate in $\mathrm{H}_{2} \mathrm{O}_{2}$ for $1 \mathrm{~h}$, which is more effective in eliminating the background [47]. An additional pretreatment with sodium permanganate followed by oxalic acid [114] (initially intended for melanin bleaching [71]) prior to this lanthanum nitrate/sodium acetate treatment of Gallyas [47] was found highly effective in eliminating the background and is currently in use as the standard in our laboratory. The background staining is practically negligible because the expected target staining is usually obtained before long and this untoward background reaction is generally slow. Similar oxidative pretreatments are not beneficial for other silver-staining methods [68]. The subsequent gold toning is essentially similar to that for Bodian method.

Compared with Bodian and Bielschowsky methods, Gallyas method is characterized by its stability, reproducibility and ability to visualize abundant deposits [15]. Moreover, normal structures are not stained in practice. This allows easy and reproducible examination especially when looking for pathological deposits such as NFTs or glial cytoplasmic inclusions (GCIs) of multiple system atrophy (MSA) [90, 119]. However, amyloid deposits are hardly visualized and it is usually neuritic components of SPs that are labeled by Gallyas method.

\section{Campbell-Switzer method}

The initial impregnation solution of Campbell-Switzer method contains pyridine (Table 1). Pyridine was initially used for tissue fixation. Bielschowsky [8] used pyridine for postfixation of formaldehyde-fixed frozen sections and 
found an enhanced selectivity. In order to avoid instability and risk of explosion of the ammoniacal silver solution, Hicks [57] added pyridine (4.4 vol\%) to the silver nitrate solution $(0.55 \%)$ buffered with potassium carbonate at alkaline range. Thereafter, Campbell et al. [21] combined this silver-pyridine $(2.5$ vol\%)-carbonate solution with the physical developer invented by Gallyas and found that this staining is not only highly sensitive in detecting SPs and NFTs in AD brains but also as stable and reproducible as Gallyas method. The solution was later modified by increasing the concentration of pyridine up to $8.7 \%$ [13] and was found to be equally very powerful in detecting $\alpha$ synuclein-related pathologies [101]. Gallyas demonstrated that the presence of pyridine markedly decreases the amount of silver, either metallic or reducible, attached to the section [42]. This is compatible with the observation that pyridine enhances the selectivity of Bielschowsky method [8] and that a higher concentration of pyridine is associated with reduced background [21]. Because the standard pretreatment (potassium permanganate/oxalic acid followed by lanthanium nitrate) for Gallyas method does not affect the results obtained with Campbell-Switzer method and there are essentially no differences between the physical developers used for Gallyas and Campbell-Switzer methods, the only significant difference between them is the silver solutions [86]. The reason for these differences between Gallyas and Campbell-Switzer methods remains unexplained.

\section{Enhancement of silver staining by methenamine}

Methenamine has been used for histological demonstration of glycogen and mucin [52]. Periodic acid-methenamine silver stain has been used for staining of the basement membrane of the glomeruli [61]. Dekura et al. [30] first described the use of the methenamine-silver solution for neurofibrils without prior periodic acid. Further modification of this methenamine-silver method was found highly sensitive in detecting SPs, which is equivalent to immunohistochemistry for amyloid $\beta$-protein $(\mathrm{A} \beta)[1,53]$. Interestingly, formic acid pretreatment, which enhances immunoreactivity for $A \beta$ of SPs, completely diminishes argyrophilia of SPs by the methenamine-silver method or congophilia, suggesting that the methenamine-silver method detects specific structures, such as $\beta$-pleated sheets of amyloid, which are destroyed by formic acid [130]. It also stains perivascular amyloid but fails to stain Kuru plaque of Gerstmann-Sträussler-Scheinker disease [130] and renal deposits of amyloid [53]. This is in agreement with the early speculation by Seki [102] that factors other than direct affinity of silver to some tissue elements, such as the quantity of intervening colloid or packing density of tissue components are also responsible for final results of the silver staining. The paucity of normal neurofibrils with this method is helpful in delineating SPs on electron microscopy [76]. It is then possible to detect even sparse aggregations of amorphous, often ramified structures with fine granular silver deposits, suggesting that this highly sensitive method is capable of detecting the earliest stage of $A \beta$ deposition [58]. Although the mechanism of lesion-specific enhancement remains unknown, introduction of $5 \%$ of methenamine in the silver proteinate solution of Bodian method is similarly effective in enhancing the selective affinity to SPs and NFTs [65]. It is interesting that this methenamine-silver method exhibits more affinity to Pick bodies when they are extracellular [89], while intracellular counterparts are more readily stained by conventional Bodian method even with electron microscopy [91].

\section{Pretreatment with microwave}

A microwave treatment of sections in silver solution was first reported by Brinn [19]. This treatment causes an increase in molecular movements across the tissue. This presumably facilitates quicker silver impregnation. At the same time, shortened incubation time for impregnation and activated molecular movements may minimize precipitate formation. Microwave activation of silver impregnation for SPs and NFTs was first introduced in order to apply King's method [63] (originally designed for frozen sections) to paraffin-embedded sections [74]. Microwave activation is effective with Bielschowsky [23] and the methenamine silver [36] methods in shortening the incubation time for silver impregnation and eliminating the background. Although the elevated temperature may partly explain the improvements of these silver-staining methods, it has also been reported that a lowered temperature at $5^{\circ} \mathrm{C}$ for initial impregnation in 20\% silver nitrate for Yamamoto-Hirano's modification of Bielschowsky method gives more reproducible results in visualizing a larger number of diffuse plaques [73].

\section{Molecular species detected by silver staining}

It is interesting to identify isolated molecular species detected by silver-staining methods, but these attempts have been so far limited. Gambetti et al. used Bodian method and anti-neurofilament antibodies in parallel to stain an extract from rat spinal cord electrophoresed on the SDS-polyacrylamide gel. The typical triplet of 200, 145 and $68 \mathrm{kDa}$ identified on the Bodian-stained gel is in agreement with the neurofilament triplet identified on the counterpart stained with anti-neurofilament antibodies [48]. 
They confirmed similar parallelism in other vertebrate and nonvertebrate species as well [94]. Additional less intense bands at a lower molecular weight range may correspond to tubulin or degradation products of neurofilament proteins [4]. Because these findings have been obtained with normal nerve tissue, further analyses based on brains containing pathological lesions are mandatory to identify components responsible for their argyrophilia on the pathological lesions. Iqbal et al. [60] used Gallyas method to stain the SDS-gel after electrophoresis of the paired helical filament (PHF) fraction extracted from AD brain. The electrophoretic mobilities of the Gallyas-positive bands correspond to those of PHF tau (intense), normal tau (less intense) and high molecular weight MAPs (weak). The absence of Gallyas-positive bands in the range of neurofilament proteins is in sharp contrast with the Bodian-positive triplet of neurofilament [48]. This difference may explain why Gallyas method is practically free from the staining of normal structures such as neuronal soma and neurites. This discrepancy suggests that molecular species detected by different silverstaining methods can be completely different, even though both of the methods label apparently identical structures such as NFTs on histological sections. Because several silver-staining methods have been modified to stain SDS gels $[62,78,107]$, it may be possible to identify molecular species, either normal or specific to some pathological structures, which have a selective affinity to some silver-staining methods. Because extracted proteins electrophoresed on gel are necessarily modified or significantly degenerated, this approach may be fruitful only if the disease-specific nature, if detectable with silver-staining methods, is readily represented by a single isolated molecule even when it is modified for electrophoresis.

\section{Quantitative comparison of silver-staining methods and immunohistochemistry}

Even after the introduction of immunohistochemistry, modification of classic silver-staining methods or invention of new silver-staining methods have been attempted $[11,25$, $26,98]$. Two major deposits targeted by these silver-staining methods are undoubtedly NFTs and SPs of AD [31, 32, $79,82]$. Different silver-staining methods $[24,27,31,54$, $56,67,99,124,125,127]$ have been compared quantitatively for their relative ability to detect NFTs or SPs, as represented in Fig. 2 [56]. In respect to detection of NFTs, these comparative studies are in agreement that Gallyas method is most powerful, followed by one of the three methods: modified Bielschowsky, Bodian or Cross methods. The methenamine-silver method is rated as least powerful for NFTs. There are two studies, each claiming a superior or equivalent number of NFTs detected with
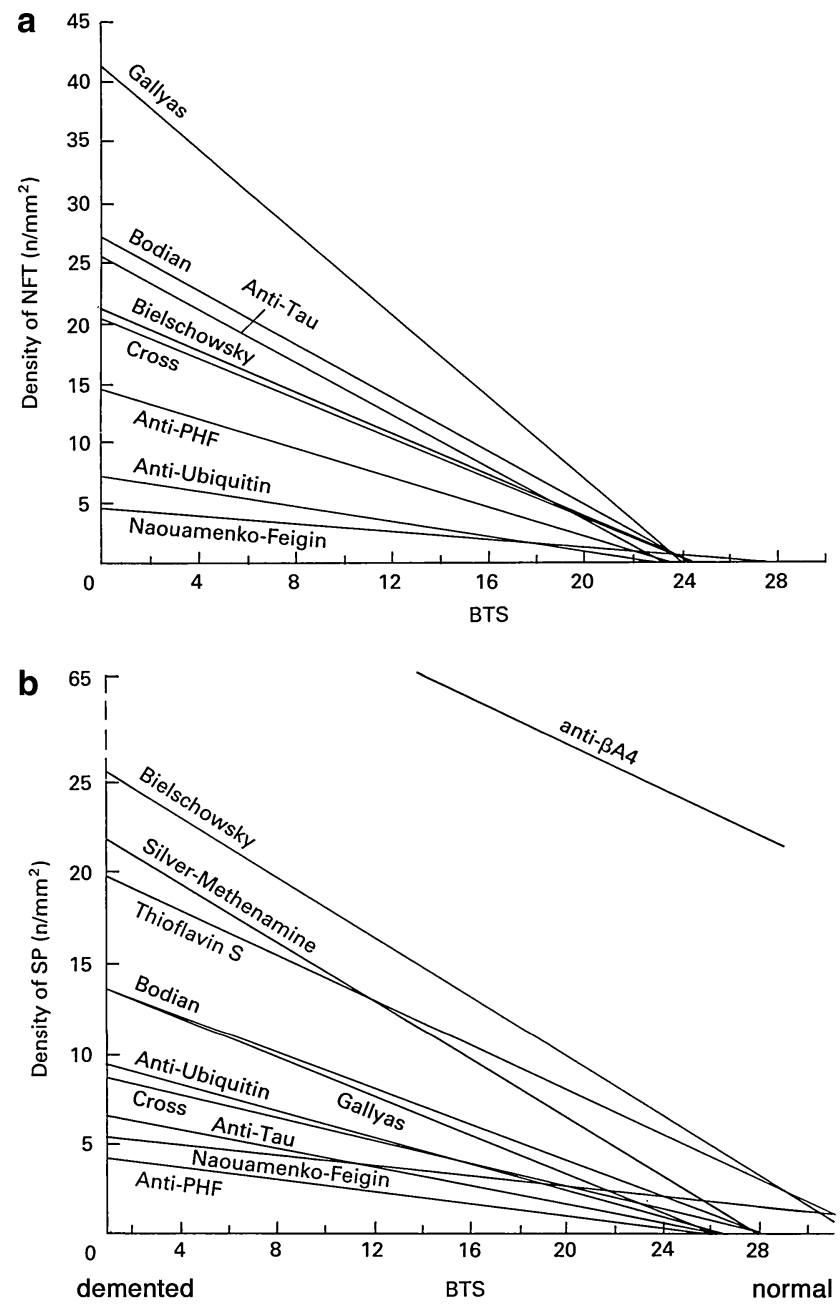

Fig. 2 Density of NFTs (a: upper panel) and that of SPs (b: lower panel) as a function of intellectual status evaluated by the Blessed Test Score. Both are dependent on the staining methods. Reproduced with permission [56]

Reusche method [99] or with the nickel peroxidase method [27] compared with Gallyas method. This rating of putative sensitivity, however, is not shared when SPs are examined. SPs are well stained by modified Bielschowsky [125, 131], the methenamine-silver [1], periodic acid methenamine silver [128] or thioflavin-S [124] to similar extent. Bodian method is less powerful in detecting SPs, especially diffuse deposits [54, 56, 67, 127, 128]. Gallyas, Cross [56, 67] and Garvey [27] methods are least powerful in detecting SPs.

It is needless to say that a more sensitive detection is preferable in order to identify specific deposits in more detail with ease. However, if one deals with clinicopathological correlation of a disease such as $\mathrm{AD}$, where symptoms and deposits are more or less quantifiable under the background of normal aging, it is possible to identify a threshold of the deposits with any of the methods that may correlate with a threshold of the cognitive decline [118]. For this purpose, stability and reproducibility of the method 
are preferable [31-33, 54, 79-82]. Reliable agreement between the conventional Braak staging of neurofibrillary pathology based on Gallyas method [17] and the revised protocol based on AT8 immunohistochemistry [18] is a good example. On the other hand, if one is looking for specific pathological deposits, such as Pick bodies, GCIs or astrocytic plaques not observed in normal aging [118], specificity is the primary concern for the staining method. Some of these comparative studies included immunohistochemistry for tau $[31,56,99]$, A $\beta[27,54,56,99,124$, 127], ubiquitin [56] or neurofilament [24] for comparison with silver-staining methods. Some studies with highly sensitive $A \beta$ immunohistochemistry demonstrated that it visualizes a larger number of deposits than with conventional silver-staining methods $[1,56]$. This suggests that amorphous $A \beta$ deposition, not identified by conventional silver-staining methods, represents the earliest stage of $A \beta$ deposition [59, 95, 129]. On the other hand, it is noteworthy that none of the series found any quantitative superiority of immunohistochemistry in identifying NFTs over the bestpossible silver-staining method. For example, it is rather frequent that pathological structures in the form of NFT do not exhibit tau-like immunoreactivity any more, while most of them are stained by some silver-staining methods or fluorochromes [116]. This is explained if some tau epitopes in NFTs are truncated out during their evolution from pretangle to extracellular NFTs [12, 116]. Furthermore, immunohistochemical detection is highly dependent on the antibody and experimental procedures. These technical uncertainties make it difficult to place immunohistochemical detection as a standard for neuropathological diagnosis, especially when the quantity of deposits is of primary importance as with the histological diagnostic criteria for $\mathrm{AD}[32,79]$. It is needless to say that the molecular specificity of immunohistochemistry is a potentially reliable and logical way in order to sort different diseases under the diagnostic flags of molecules. This highly specific detection by pinpointing a single target molecule is, however, "a very restricted, monochromic view" as pointed out by Switzer [108]". Identification of the disease-specific molecular species and their reliable probes applicable to histological sections are desirable. Even if this kind of probes are available, immunohistochemical procedures are also difficult to standardize and much more costly than most of silver-staining methods [67]. Immunohistochemistry is highly costly especially when staining large histological samples, for example, hemispheric sections of human brain. Moreover, penetration of antibodies is sometimes not sufficient when staining thick histological sections. Silver-staining methods are more readily applicable even to large and thick histological sections [13]. It is of note as well that archival materials preserved for a long period, especially in formaldehyde, sometimes fail to exhibit immunoreactivity. It is frequently possible to visualize the lesions with silverstaining methods even in these archival materials $[13,123]$.

\section{Qualitative representation by silver-staining methods}

As summarized in the previous section, differences in the quantity of argyrophilic $\mathrm{AD}$ lesions are dependent on the silver-staining method. As has been considered frequently, it may be "the sensitivity" of a staining method that governs the quantity of the argyrophilic lesions. This is probably in agreement with our general impression that "modified Bielschowsky method is more powerful than Bodian method in detecting AD lesions." [131] This interpretation is based on the assumption that so-called "argyrophilia" is a homogeneous phenomenon regardless of the staining method and the nature of lesion, which is not the case. For example, Gallyas method is one of the most "sensitive" methods in detecting NFTs and neuropil threads in AD, while it is far less "sensitive" in detecting SPs. This empirical but evident discrepancy is not readily explained by a mere difference in the "sensitivity" of the methods and rather indicates that argyrophilic properties are dependent not only on the staining methods but also on the target lesions. Careful neuropathologists have been aware of these "method- and lesion-dependent natures" of various argyrophilic deposits in neurodegenerative diseases [16, 85, 96]. Table 2 provides a summary of the heterogeneity of argyrophilia according to the staining method and the lesion. Gallyas method is one of the most "sensitive" silver-staining method that clearly labels NFTs of AD, as well as neuronal and glial lesions of corticobasal degeneration/progressive supranuclear palsy (CBD/PSP) [113, 120]. This "sensitive" method, however, fails to stain Pick bodies, another distinct tau-positive deposit $[16,85,96,117,118]$. On the other hand, Campbell-Switzer method labels Pick bodies but not CBD/PSP-related lesions [120, 122]. This reversed and complementary profile with Gallyas and Campbell-Switzer methods is hardly explained by a superior sensitivity of Gallyas method over Campbell-Switzer method or vise versa. These discrepancies between Gallyas and CampbellSwitzer methods are readily demonstrable if mirror section pairs are initially fluorolabeled and one of the section pair is subsequently stained with Gallyas and the counterpart with Campbell-Switzer method, as shown in Fig. 3. It is now known that molecular species of tau are different according to the disease-specific lesions. Namely, Pick bodies are usually composed of three-repeat $(3 \mathrm{R})$ tau, while $\mathrm{CBD} /$ PSP-related tau pathologies are positive for four-repeat (4R) tau [28]. Because Pick bodies are usually positive with Campbell-Switzer method but negative with Gallyas method (Fig. 3a-d), one of the possible interpretations is that 3R tau deposits, such as Pick bodies, exhibit argyro- 
Table 2 Heterogeneity of argyrophilia according to disease and method

\begin{tabular}{|c|c|c|c|c|}
\hline Staining methods & Campbell-Switzer & Bodian & Bielschowsky & Gallyas \\
\hline Pick bodies $[16,85,96,117,118]$ & ++ & ++ & ++ & - \\
\hline AD-NFTs & ++ & ++ & ++ & ++ \\
\hline Down-NFTs & ++ & ++ & ++ & ++ \\
\hline DNTC-NFTs & ++ & ++ & ++ & ++ \\
\hline PSP/CBD-neurons & - & $+^{\mathrm{a}}$ & $+{ }^{\mathrm{b}}$ & ++ \\
\hline PSP/CBD-glia & - & $\pm^{c}$ & $t^{\mathrm{b}}$ & ++ \\
\hline Argyrophilic grains $[14,77,112]$ & - & + & + & ++ \\
\hline AD-SPs & ++ & ++ & ++ & + \\
\hline AD-diffuse deposits & ++ & - & \pm & - \\
\hline Lewy bodies $[75,119]$ & ++ & + & ++ & - \\
\hline GCIs $[87,90]$ & ++ & + & + & ++ \\
\hline
\end{tabular}

$A D$ Alzheimer's disease, NFTs neurofibrillary tangles, $D N T C$ diffuse NFTs with calcification, $P S P$ progressive supranuclear palsy, $C B D$ corticobasal degeneration, $S P$ senile plaques, $G C I s$ glial cytoplasmic inclusions, ++ : easily recognizable, + : positive, \pm : questionably positive, - : negative

${ }^{a}$ Positive in PSP cases [55] but less evident in CBD cases [67, 109, 115]

b Not consistent[97], positive in some cases, not appropriate for histological examination

c The argyrophilia (Bodian) is less evident in glia than in neurons, especially in CBD cases

philia with Campbell-Switzer but not with Gallyas method. In contrast, the lesions of $4 \mathrm{R}$ tauopathy (CBD/PSP and argyrophilic grain disease $[110,111])$ are related to the argyrophilia with Gallyas but not with Campbell-Switzer method (Fig. 3i-1) [120]. Indeed, NFTs of AD, Down syndrome, and diffuse neurofibrillary tangles with calcification, all containing both $3 R$ and $4 R$ isoforms, similarly exhibit argyrophilia with either method (Fig. 3e-h) [120-122]. It is interesting that Gallyas-positive Pick-like bodies were found to be positive for $4 R$ tau but sparsely positive for $3 R$ tau [132], which corroborates the empirical correlation between 4R tau and Gallyas method. Moreover, in one of the largest studies on sporadic and familial frontotemporal degeneration with tau-positive deposits, "Pick bodies" defined as "inclusions stained by Bielschowsky but not by Gallyas" were found in 17 cases. All exhibited the immunoreactivity with a 3R-tau specific antibody as well as $3 \mathrm{R}$ tau predominance in tau biochemistry except for a single case with E342V mutation in the tau gene [29]. This particular case is characterized by diffuse cytoplasmic tau staining in neurons and PHF-like electron microscopic features [72], both of which are not typical of Pick bodies. In another sporadic case, Bodian-positive inclusions positive for $4 \mathrm{R}$ but not $3 \mathrm{R}$ tau have electron microscopic features with twisted ribbon distinct from those of typical Pick bodies [83]. These apparent heterogeneities necessitate a revision of the classical definition of Pick bodies "round argyrophilic, tau-positive inclusions in neuronal cytoplasm", because argyrophilic features and tau-species are both heterogeneous in this classical category. Among argryophilic cytoplasmic inclusions in neuron, it seems that those positive for 3R-tau [84] and those negative with Gall- yas method [29] are correlated to form a cluster to be defined as Pick bodies. Argyrophilia with the CampbellSwitzer method [122] is helpful for more precise and straightforward definition of Pick body. In contrast with Gallyas and Campbell-Switzer methods, argryrophilia with Bielschowsky and Bodian methods are less dependent on the target lesions (Table 2). It is noteworthy that tau-positive cortical lesions of PSP are silver-stained with Bodian method [55], while those of CBD frequently fail to exhibit argyrophilia with Bodian method $[69,109,115]$ especially in the cerebral cortices. Although possible biochemical distinctions of tau between CBD and PSP have been described $[3,104]$, it remains to be clarified how these biochemical differences are related to these morphological differences between CBD and PSP. Another possible limitation is related to the observations that the expression of tau-isoforms is not homogeneous throughout the brain [51, 126]. Because most of the data on tau-positive deposits summarized in Table 2 and Fig. 3 have been obtained on cerebral cortices, it remains to be determined whether these findings are similarly applicable to extracortical areas such as the brainstem. Yoshida [132] demonstrated that 3R tau immunoreactivity is represented in the brainstem even in so-called $4 \mathrm{R}$ tauopathies such as CBD/PSP. Because neurofibrillary pathologies related to $\mathrm{AD}$ or aging are sometimes found in the brainstem nuclei and usually positive for both $3 R$ and $4 R$ isoforms, it remains to be clarified whether the presence of $3 R$ tau in brainstem nuclei in CBD/PSP is ascribed to tauopathies independent of normal or accelerated aging.

Interestingly, argyrophilic differentiation with Gallyas and Campbell-Switzer methods is not limited to tau- 

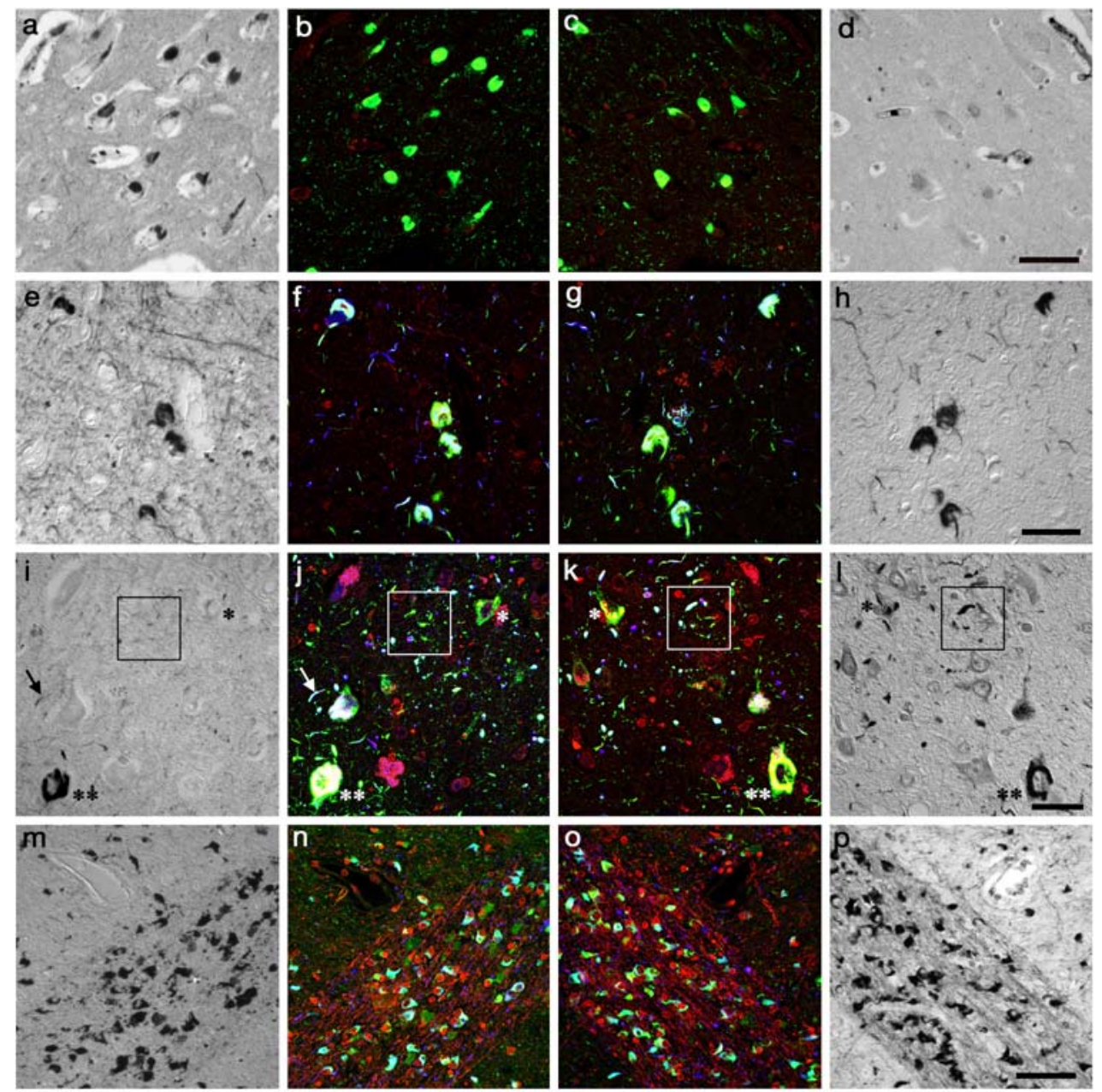

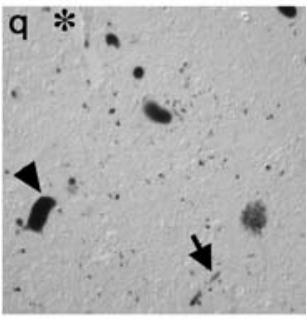

Campbell-Switzer

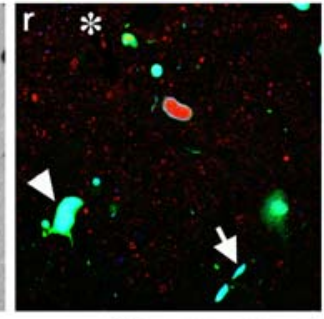

Fluorescence (mirror sections)

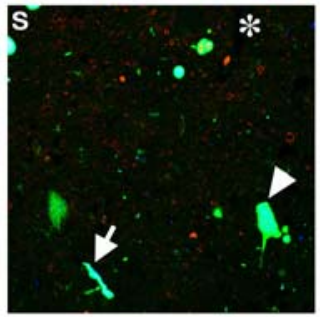

[121]. This staining profile is shared with NFTs of AD [122]. Argyrophilic grains (i-l, in the square) and tau-positive neurons are present ( $\mathbf{j}$, k) in the parahippocampal gyrus. The tau-positive neuron (double asterisk) and neuropil thread (arrow) stained with both CS and GAL are similar to those observed in DNTC $(\mathbf{e}-\mathbf{h})$, representing neurofibrillary pathology. Grains and pretangle neuron (single asterisk) are stained with GAL (l) but not with CS (i) in contrast. This staining profile is shared with cortical lesion of corticobasal degeneration/progressive supranuclear palsy [120]. Glial cytoplasmic inclusions $(\mathbf{m}-\mathbf{p})$ in the putamen, positive for $\alpha$-synuclein $(\mathbf{n}, \mathbf{o})$, are stained with both CS (m) and GAL (p) [119]. Lewy bodies (arrowhead, q-t) and Lewy neurites (arrow, $\mathbf{q}-\mathbf{t}$ ) in the dorsal motor nucleus of vagus, positive for $\alpha$ synuclein $(\mathbf{r}, \mathbf{s})$, are stained with CS (q) but not with GAL (t) [119]. The same blood vessel is indicated with asterisk $(\mathbf{q}-\mathbf{t})$. Bar $50 \mu \mathrm{m}(\mathbf{a}-$ $\mathbf{d}, \mathbf{m}-\mathbf{p}, \mathbf{q}-\mathbf{t})$, bar $30 \mu \mathrm{m}(\mathbf{e}-\mathbf{l})$. Reproduced with permission. a-d [122]; e-l [121]; m-t [119] 
positive deposits. Both of the two staining methods label GCIs of MSA (Fig. 3m-p) [119]. Although GCIs are positive for some restricted tau epitopes [106], their major constituent is now considered to be $\alpha$-synuclein. Indeed, Campbell-Switzer method clearly labels Lewy bodies and their neurites, another representative deposits of $\alpha$-synuclein [13]. It is interesting that Gallyas method fails to label Lewy bodies and Lewy neurites (Fig. 3q-t). Therefore, these two silver-staining methods, when performed in parallel, provide staining profiles that may allow qualitative distinction between GCIs and Lewy bodies [119]. Because no biochemical markers that enable distinction between Lewy bodies and GCIs are not readily available, these differential profiles in silver staining provides an additional qualitative distinction possibly linked to underlying molecular differences.

At present, these empirical distinctions are useful for diagnostic differentiation but await further explanations preferably at molecular or ultrastructural level. These distinctive features of silver-staining profiles may be explained if (1) molecular species specific to each subcategory (for example, 3R vs. 4R tau or LBs vs. GCIs) has different affinity to each silver-staining method; (2) some disease-specific ultrastructures of pathological deposit are related to each staining profile; (3) other independent molecules in these deposits play some roles in characterizing the pathological deposits so that distinctive silver-staining profiles are engendered; or (4) some disease-specific conformational changes are related to a silver-staining profile specific to a disease. Because Campbell-Switzer and Gallyas methods are useful in discriminating not only tau-positive deposits but also $\alpha$-synuclein-positive deposits even with their molecular and ultrastructural diversities, it is plausible that factors such as (3) or (4) shared by tau-positive deposits and synuclein-positive deposits may play roles, at least partly, in these discriminating abilities with these silver-staining methods.

\section{Conclusions and perspective}

Even with my limited experience on these silver-staining methods, it is apparent that the argyrophilic profiles are dependent not only on the silver-staining method but also on the target lesion. This indicates that "argyrophilia" is highly heterogeneous. However, this heterogeneity is not capricious but rather representative of some disease-specific aspects. The apparent empirical correlations between the argyrophilic profiles and the disease-specific pathological structures are useful in sorting these neurodegenerative disorders. Combined silver-staining profiles, for example with Gallyas and Campbell-Switzer methods, are more convenient than modified Bielschowsky and Bodian method for sorting different disease-specific lesions. Although these staining profiles are discussed in this review as if disease-specific, more detailed comparison at cellular level demonstrated that they can be different from cell to cell even in the same section (intercellular differences) [117]. Furthermore, staining profiles are heterogeneous even in a single cell (intracellular differences) [49]. These silver-staining and immunohistochemical profiles are much more informative especially when combined together than when used in isolation because such combination is not a mere summation of the results obtained with different methods. It will rather provide a more stereoscopic and multifaceted view, which may allow more precise morphological delineation of disease-specific deposits. In my view, comments such as "Silver-staining methods are unreliable, nonreproducible, nonscientific and out-dated." are now out-dated. Awareness of their utility and reliability of silverstaining methods, different from immunohistochemistry, may facilitate diagnosis and accelerate research, which hopefully clarifies molecular basis of each silver-staining method and improves our understanding of diseases.

Acknowledgments Everlasting encouragement by honorary professor Dr. Hajime Mannnen (Department of Neuroanatomy, Tokyo Medical and Dental University) is highly acknowledged. I am grateful to Dr. Kuniaki Tsuchiya (Department of Pathology and Laboratory Medicine, Tokyo Metropolitan Matsuzawa Hospital), Dr. Saburo Yagishita (Department of Pathology, Kanagawa Rehabilitation Hospital) and Dr. Toshio Mizutani (Department of Pathology, Tokyo Metropolitan Neurological Hospital) for their kind help. Excellent technical expertise of Ms. Ayako Nakamura (Department of Neuropathology, Tokyo Metropolitan Institute for Neuroscience) is highly appreciated. This work is supported in part by the grant to "Three-dimension project (TU)" from the Tokyo Metropolintan Organization for Medical Research.

\section{References}

1. Allsop D, Haga SI, Haga C, Ikeda SI, Mann DM, Ishii T (1989) Early senile plaques in Down's syndrome brains show a close relationship with cell bodies of neurons. Neuropathol Appl Neurobiol 15:531-542

2. Alzheimer A (1911) Über eigenartige Krankheitsfälle des späten Alters. Zeitschr Gesamte Neurol Psychiat 1911:365-385

3. Arai T, Ikeda K, Akiyama H, Nonaka T, Hasegawa M, Ishiguro K, Iritani S, Tsuchiya K, Iseki E, Yagishita S, Oda T, Mochizuki A (2004) Identification of amino-terminally cleaved tau fragments that distinguish progressive supranuclear palsy from corticobasal degeneration. Ann Neurol 55:72-79

4. Autilio-Gambetti L, Crane R, Gambetti P (1986) Binding of Bodian's silver and monoclonal antibodies to defined regions of human neurofilament subunits: Bodian's silver reacts with a highly charged unique domain of neurofilaments. J Neurochem 46:366-370

5. Baker J (1966) Cytological technique. The principles underlying routine methods. Chapman and Hall, London

6. Beech R, Davenport H (1933) The Bielschowsky staining technic. A study of the factors influencing its specificity for nerve fibers. Stain Technol 8:11-30 
7. Berrios G (1990) Alzheimer's disease: a conceptual history. Int J Geriatr Psychiatry 5:355-365

8. Bielschowsky M (1908) Eine Modifikation meines Silverimprägnationsverfahrens zur Darstellung der Neurofibrillen. J für Psychologie Neurologie 12:135-137

9. Bodian D (1936) A new method for staining nerve fibers and nerve endings in mounted paraffin sections. Anat Res 65:89-97

10. Bodian D (1937) The staining of paraffin sections of nervous tissues with activated protargol. The role of fixatives. Anat Res 69:153-162

11. Bolle L, Maurer B, Janzer RC (1992) A modified HortegaGlobus stain is superior to Bielschowsky and Bodian stains for demonstrating neuritic plaques. Biotech Histochem 67:82-87

12. Bondareff W, Harrington CR, Wischik CM, Hauser DL, Roth M (1995) Absence of abnormal hyperphosphorylation of tau in intracellular tangles in Alzheimer's disease. J Neuropathol Exp Neurol 54:657-663

13. Braak E, Braak H (1999) Silver staining method for demonstrating Lewy bodies in Parkinson's disease and argyrophilic oligodendrocytes in multiple system atrophy. J Neurosci Methods 87:111-115

14. Braak H, Braak E (1987) Argyrophilic grains: characteristic pathology of cerebral cortex in cases of adult onset dementia without Alzheimer changes. Neurosci Lett 76:124-127

15. Braak H, Braak E, Ohm T, Bohl J (1988) Silver impregnation of Alzheimer's neurofibrillary changes counterstained for basophilic material and lipofuscin pigment. Stain Technol 63:197-200

16. Braak H, Braak E (1989) Cortical and subcortical argyrophilic grains characterize a disease associated with adult onset dementia. Neuropathol Appl Neurobiol 15:13-26

17. Braak H, Braak E (1991) Neuropathological stageing of Alzheimer-related changes. Acta Neuropathol (Berl) 82:239-259

18. Braak H, Alafuzoff I, Arzberger T, Kretzschmar H, Del Tredici K (2006) Staging of Alzheimer disease-associated neurofibrillary pathology using paraffin sections and immunocytochemistry. Acta Neuropathol (Berl) 112:389-404

19. Brinn N (1983) Rapid metallic histological staining using the microwave oven. J Histotechnol 6:125-129

20. Bruchez M Jr, Moronne M, Gin P, Weiss S, Alivisatos AP (1998) Semiconductor nanocrystals as fluorescent biological labels. Science 281:2013-2016

21. Campbell S, Switzer R, Martin T (1987) Alzheimer's plaques and tangles: a controlled and enhanced silver staining method. Soc Neurosci Abst 13:678

22. Chan WC, Nie S (1998) Quantum dot bioconjugates for ultrasensitive nonisotopic detection. Science 281:2016-2018

23. Churukian C, Kazee A, Lapham L, Eskin T (1992) Microwave modification of Bielschowsky silver impregnation method for diagnosis of Alzheimer's disease. J Histotechnol 15:299-302

24. Connolly AA, Anderton BA, Esiri MM (1987) A comparative study of a silver stain and monoclonal antibody reactions on Alzheimer's neurofibrillary tangles. J Neurol Neurosurg Psychiatry 50:1221-1224

25. Cross RB (1982) Demonstration of neurofibrillary tangles in paraffin sections: a quick and simple method using a modification of Palmgren's method. Med Lab Sci 39:67-69

26. Cullen KM (1994) A novel nickel avidin-biotin-peroxidase method for histochemical visualization of neurofibrillary tangles, senile plaques, and neuropil threads. J Histochem Cytochem 42:1383-1391

27. Cullen KM, Halliday GM, Cartwright H, Kril JJ (1996) Improved selectivity and sensitivity in the visualization of neurofibrillary tangles, plaques and neuropil threads. Neurodegeneration 5:177187

28. de Silva R, Lashley T, Gibb G, Hanger D, Hope A, Reid A, Bandopadhyay R, Utton M, Strand C, Jowett T, Khan N, Anderton B,
Wood N, Holton J, Revesz T, Lees A (2003) Pathological inclusion bodies in tauopathies contain distinct complements of tau with three or four microtubule-binding repeat domains as demonstrated by new specific monoclonal antibodies. Neuropathol Appl Neurobiol 29:288-302

29. de Silva R, Lashley T, Strand C, Shiarli AM, Shi J, Tian J, Bailey KL, Davies P, Bigio EH, Arima K, Iseki E, Murayama S, Kretzschmar H, Neumann M, Lippa C, Halliday G, MacKenzie J, Ravid R, Dickson D, Wszolek Z, Iwatsubo T, Pickering-Brown SM, Holton J, Lees A, Revesz T, Mann DM (2006) An immunohistochemical study of cases of sporadic and inherited frontotemporal lobar degeneration using 3R- and 4R-specific tau monoclonal antibodies. Acta Neuropathol (Berl) 111:329-340

30. Dekura Z, Suzuki K, Sasaki N, Yamamoto E, Goto Y, Oka T, Ohami H (1985) New staining method for neurofibrils with methenamine silver. Pathol Clin [Byori to Rinsyo (in Japanese)] 3:340-341

31. Duyckaerts C, Brion JP, Hauw JJ, Flament-Durand J (1987) Quantitative assessment of the density of neurofibrillary tangles and senile plaques in senile dementia of the Alzheimer type. Comparison of immunocytochemistry with a specific antibody and Bodian's protargol method. Acta Neuropathol (Berl) 73:167-170

32. Duyckaerts C, Delaère $P$, Hauw JJ, Abbamondi-Pinto AL, Delaère, Sorbi S, Allen I, Brion JP, Flament-Durand J, Duchen L, Kauss J et al (1990) Rating of the lesions in senile dementia of the Alzheimer type: concordance between laboratories. A European multicenter study under the auspices of EURAGE. J Neurol Sci 97:295-323

33. Duyckaerts C, Bennecib M, Grignon Y, Uchihara T, He Y, Piette F, Hauw JJ (1997) Modeling the relation between neurofibrillary tangles and intellectual status. Neurobiol Aging 18:267-273

34. Fajersztajn J (1901) Ein neues Silberimprägnationsverfahren als Mittel zur Färbung der Axencylinder. Neurologisches Centralblatt 20:98-107

35. Feigin I, Naoumenko J (1976) Some chemical principles applicable to some silver and gold staining methods for neuropathological studies. J Neuropathol Exp Neurol 35:495-507

36. Flowers D, Harasty J, Halliday G, Kril J (1996) Microwave modification of the methenamine silver technique for the demonstration of Alzheimer-type pathology. J Histotechnol 19:33-38

37. Gallyas F (1970) Silver staining of collagen and reticulin fibres and crebral capillaries by means of physical development. J Microsc 91:119-124

38. Gallyas F (1970) Silver staining of fibrous neuroglia by means of physical development. Acta Neuropathol (Berl) 16:39-43

39. Gallyas F (1970) Silver staining of micro- and oligodendroglia by means of physical development. Acta Neuropathol (Berl) 16:3538

40. Gallyas F (1971) A principle for silver staining of tissue elements by physical development. Acta Morphol Acad Sci Hung 19:5771

41. Gallyas F (1971) Silver staining of Alzheimer's neurofibrillary changes by means of physical development. Acta Morphol Acad Sci Hung 19:1-8

42. Gallyas F (1979) Factors affecting the formation of metallic silver and the binding of silver ions by tissue components. Histochemistry 64:97-109

43. Gallyas F (1979) Light insensitive physical developers. Stain Technol 54:173-176

44. Gallyas F (1980) Chemical nature of the first products (nuclei) of the argyrophil staining. Acta Histochem 67:145-158

45. Gallyas F (1982) Physico-chemical mechanism of the argyrophil I reaction. Histochemistry 74:393-407

46. Gallyas F (1982) Suppression of the argyrophil III reaction by mercapto compounds (a prerequisite for the intensification of certain histochemical reactions by physical developers). Acta Histochem 70:99-105 
47. Gallyas F, Wolff JR (1986) Metal-catalyzed oxidation renders silver intensification selective. Applications for the histochemistry of diaminobenzidine and neurofibrillary changes. J Histochem Cytochem 34:1667-1672

48. Gambetti P, Autilio Gambetti L, Papasozomenos SC (1981) Bodian's silver method stains neurofilament polypeptides. Science 213:1521-1522

49. Garcia-Sierra F, Hauw JJ, Duyckaerts C, Wischik CM, LunaMuñoz J, Mena R (2000) The extent of neurofibrillary pathology in perforant pathway neurons is the key determinant of dementia in the very old. Acta Neuropathol (Berl) 100:29-35

50. Garven HS, Gairns FW (1952) The silver diamine ion staining of peripheral nerve elements and the interpretation of the results: with a modification of the Bielschowsky-Gros method for frozen sections. Q J Exp Physiol Cogn Med Sci 37:131-142

51. Gibb GM, de Silva R, Revesz T, Lees AJ, Anderton BH, Hanger DP (2004) Differential involvement and heterogeneous phosphorylation of tau isoforms in progressive supranuclear palsy. Brain Res Mol Brain Res 121:95-101

52. Gomori H (1946) A new histochemical test for glycogen and mucin. Am J Clin Pathol 16:177-179

53. Haga C, Ikeda K, Iwabuchi K, Akiyama H, Kondoh H, Kosaka K (1994) Methenamine-silver staining: a simple and sensitive staining method for senile plaques and neurofibrillary tangles. Biotech Histochem 69:295-300

54. Halliday G, Flowers D, Baum L (1994) Analysis of staining methods for different cortical plaques in Alzheimer's disease. Acta Neuropathol (Berl) 87:174-186

55. Hauw JJ, Verny M, Delaère $\mathrm{P}$, Cervera $\mathrm{P}, \mathrm{He} \mathrm{Y}$, Duyckaerts $\mathrm{C}$ (1990) Constant neurofibrillary changes in the neocortex in progressive supranuclear palsy. Basic differences with Alzheimer's disease and aging. Neurosci Lett 119:182-186

56. He Y, Duyckaerts C, Delaère P, Piette F, Hauw JJ (1993) Alzheimer's lesions labelled by anti-ubiquitin antibodies: comparison with other staining techniques. A study of 15 cases with graded intellectual status in ageing and Alzheimer's disease. Neuropathol Appl Neurobiol 19:364-371

57. Hicks S (1946) A rapid pyridine silver stain for nervous tissue and reticular fibers. J Lab Clin Med 31:1357-1377

58. Ikeda K, Haga C, Kosaka K, Oyanagi S (1989) Senile plaque-like structures: observation of a probably unknown type of senile plaque by periodic-acid methenamine silver (PAM) electron microscopy. Acta Neuropathol (Berl) 78:137-142

59. Ikeda S, Allsop D, Glenner GG (1989) Morphology and distribution of plaque and related deposits in the brains of Alzheimer's disease and control cases. An immunohistochemical study using amyloid beta-protein antibody. Lab Invest 60:113-122

60. Iqbal K, Braak E, Braak H, Zaidi T, Grundke-Iqbal I (1991) A silver impregnation method for labeling both Alzheimer paired helical filaments and their polypeptides separated by sodium dodecyl sulfate-polyacrylamide gel electrophoresis. Neurobiol Aging 12:357-361

61. Jones DB (1957) Nephrotic glomerulonephritis. Am J Pathol 33:313-329

62. Kerenyi L, Gallyas F (1972) A highly sensitive method for demonstrating proteins in electrophoretic, immunoelectrophoretic and immunodiffusion preparations. Clin Chim Acta $38: 465-467$

63. King L (1941) The impregnation of neurofibrils. Yale J Biol Med 14:59-68

64. Kondo H, Ikeda K, Miyazaki N (1996) The mechanism of Bodian's silver staining: effect of copper ion on silver impregnation. J Neurosci Methods 68:275-280

65. Kondoh H, Matsushita M, Kosaka K, Miyazaki N (1993) Staining senile plaques using Bodian's method modified with methenamine. Biotech Histochem 68:113-116
66. Kubie L, Davidson D (1928) The ammoniacal silver solutions used in neuropathology. Their staining properties, chemistry and methods of preparation. AMA Arch Neurol Psychiatry 19:888903

67. Lamy C, Duyckaerts C, Delaère P, Payan C, Fermanian J, Poulain V, Hauw JJ (1989) Comparison of seven staining methods for senile plaques and neurofibrillary tangles in a prospective series of 15 elderly patients. Neuropathol Appl Neurobiol 15:563578

68. Lhotka JF, Myhre BA, Combs CM (1953) Effects of oxidation on neurofibrillar argyrophilia. Stain Technol 28:101-105

69. Li F, Iseki E, Odawara T, Kosaka K, Yagishita S, Amano N (1998) Regional quantitative analysis of tau-positive neurons in progressive supranuclear palsy: comparison with Alzheimer's disease. J Neurol Sci 159:73-81

70. Liesegang R (1911) Die Kolloidchemie der histologischen Silberfärbungen. Kolloidchemische Beihefte 3:1-46

71. Lillie RD (1957) Trichoxanthin, the yellow granular pigment of guinea pig hair follicles and hairs. J Histochem Cytochem 5:346353

72. Lippa CF, Zhukareva V, Kawarai T, Uryu K, Shafiq M, Nee LE, Grafman J, Liang Y, St George-Hyslop PH, Trojanowski JQ, Lee VM (2000) Frontotemporal dementia with novel tau pathology and a Glu342Val tau mutation. Ann Neurol 48:850-858

73. Litchfield S, Nagy Z (2001) New temperature modification makes the Bielschowsky silver stain reproducible. Acta Neuropathol (Berl) 101:17-21

74. Lloyd B, Brinn N, Burger PC (1985) Silver-staining of senile plaques and neurofibrillary change in paraffin-embedded tissues. J Histotechnol 8:155-156

75. Love S, Nicoll JA (1992) Comparison of modified Bielschowsky silver impregnation and anti-ubiquitin immunostaining of cortical and nigral Lewy bodies. Neuropathol Appl Neurobiol 18:585-592

76. Makifuchi T, Watabe K, Ikuta F (1986) The study of senile plaques by the PAM staining: the detection of amyloid [abstract]. Neuropathology 7:196

77. Martinez-Lage P, Muñoz DG (1997) Prevalence and disease associations of argyrophilic grains of Braak. J Neuropathol Exp Neurol 56:157-164

78. Merril CR, Switzer RC, Van Keuren ML (1979) Trace polypeptides in cellular extracts and human body fluids detected by twodimensional electrophoresis and a highly sensitive silver stain. Proc Natl Acad Sci USA 76:4335-4339

79. Mirra SS, Heyman A, McKeel D, Sumi SM, Crain BJ, Brownlee LM, Vogel FS, Hughes JP, van Belle G, Berg L (1991) The Consortium to Establish a Registry for Alzheimer's Disease (CERAD). Part II. Standardization of the neuropathologic assessment of Alzheimer's disease. Neurology 41:479-486

80. Mirra SS, Hart MN, Terry RD (1993) Making the diagnosis of Alzheimer's disease. A primer for practicing pathologists. Arch Pathol Lab Med 117:132-144

81. Mirra SS (1997) The CERAD neuropathology protocol and consensus recommendations for the postmortem diagnosis of Alzheimer's disease: a commentary. Neurobiol Aging 18:S91-S94

82. Mirra SS (1997) Neuropathological assessment of Alzheimer's disease: the experience of the Consortium to Establish a Registry for Alzheimer's Disease. Int Psychogeriatr 9 Suppl 1:263-268; discussion 269-272

83. Motoi Y, Iwamoto H, Itaya M, Kobayashi T, Hasegawa M, Yasuda M, Mizuno Y, Mori H (2005) Four-repeat tau-positive Pick body-like inclusions are distinct from classic Pick bodies. Acta Neuropathol (Berl) 110:431-433

84. Mott RT, Dickson DW, Trojanowski JQ, Zhukareva V, Lee VM, Forman M, Van Deerlin V, Ervin JF, Wang DS, Schmechel DE, Hulette CM (2005) Neuropathologic, biochemical, and molecular 
characterization of the frontotemporal dementias. J Neuropathol Exp Neurol 64:420-428

85. Muñoz D (1999) Stains for the differential diagnosis of degenerative dementias. Biotech Histochem 74:311-321

86. Nakamura A, Shibuya K, Yagishita S, Uchihara T (2005) Distinguishing tau-positive deposits by different silver staining methods. Pathol Clin [Byori to Rinsyo (In Japanese)] 23:427-433

87. Nakazato Y, Yamazaki H, Hirato J, Ishida Y, Yamaguchi H (1990) Oligodendroglial microtubular tangles in olivopontocerebellar atrophy. J Neuropathol Exp Neurol 49:521-530

88. Newman GR, Jasani B (1998) Silver development in microscopy and bioanalysis: past and present. J Pathol 186:119-125

89. Odawara T, Iseki E, Furukawa Y, Suzuki K, Hino H, Kosaka K (2002) Degeneration of Pick bodies visualized by methenaminesilver staining and immunohistochemistry. Neuropathology 22:180-185

90. Papp MI, Kahn JE, Lantos PL (1989) Glial cytoplasmic inclusions in the CNS of patients with multiple system atrophy (striatonigral degeneration, olivopontocerebellar atrophy and Shy-Drager syndrome). J Neurol Sci 94:79-100

91. Perry G, Stewart D, Friedman R, Manetto V, Autilio-Gambetti L, Gambetti P (1987) Filaments of Pick's bodies contain altered cytoskeletal elements. Am J Pathol 127:559-568

92. Peters A (1958) Staining of nervous tissue by protein-silver mixtures. Stain Technol 33:47-53

93. Peters A (1959) Experimental studies on the staining of nervous tissue with silver proteinates. Am J Anat 93:177-195

94. Phillips LL, Autilio-Gambetti L, Lasek RJ (1983) Bodian's silver method reveals molecular variation in the evolution of neurofilament proteins. Brain Res 278:219-223

95. Probst A, Brunnschweiler H, Lautenschlager C, Ulrich J (1987) A special type of senile plaque, possibly an initial stage. Acta Neuropathol (Berl) 74:133-141

96. Probst A, Tolnay M, Langui D, Goedert M, Spillantini MG (1996) Pick's disease: hyperphosphorylated tau protein segregates to the somatoaxonal compartment. Acta Neuropathol (Berl) 92:588-596

97. Rebeiz JJ, Kolodny EH, Richardson EP Jr (1968) Corticodentatonigral degeneration with neuronal achromasia. Arch Neurol 18:20-33

98. Reusche E (1991) Silver staining of senile plaques and neurofibrillary tangles in paraffin sections. A simple and effective method. Pathol Res Pract 187:1045-1049

99. Rosenwald A, Reusche E, Ogomori K, Teichert HM (1993) Comparison of silver stainings and immunohistology for the detection of neurofibrillary tangles and extracellular cerebral amyloid in paraffin sections. Acta Neuropathol (Berl) 86:182186

100. Samuel EP (1953) The mechanism of silver staining. J Anat $87: 278-287$

101. Sandmann-Keil D, Braak H, Okochi M, Haass C, Braak E (1999) Alpha-synuclein immunoreactive Lewy bodies and Lewy neurites in Parkinson's disease are detectable by an advanced silverstaining technique. Acta Neuropathol (Berl) 98:461-464

102. Seki M (1940) Zur Theorie der histologischen Silverschwärzung. I.Gliazellendarstellung. Zeitschrift für Zellforschung und mikroskopische Anatomie 30:529-547

103. Seki M (1961) Histological methods [Soshiki Kensa Hou (in Japanese)]. Kyorin-Shoin, Tokyo

104. Sergeant N, Wattez A, Delacourte A (1999) Neurofibrillary degeneration in progressive supranuclear palsy and corticobasal degeneration: tau pathologies with exclusively "exon 10" isoforms. J Neurochem 72:1243-1249

105. Sevier AC, Munger BL (1965) Technical note: a silver method for paraffin sections of neural tissue. J Neuropathol Exp Neurol 24:130-135
106. Shibuya K, Uchihara T, Nakamura A, Ishiyama M, Yamaoka K, Yagishita S, Iwabuchi K, Kosaka K (2003) Reversible conformational change of tau2 epitope on exposure to detergent in glial cytoplasmic inclusions of multiple system atrophy. Acta Neuropathol (Berl) 105:508-514

107. Switzer RC III, Merril CR, Shifrin S (1979) A highly sensitive silver stain for detecting proteins and peptides in polyacrylamide gels. Anal Biochem 98:231-237

108. Switzer RC, III (1993) Silver staining methods: their role in detecting neurotoxicity. Ann N Y Acad Sci 679:341-348

109. Takahashi T, Amano N, Hanihara T, Nagatomo H, Yagishita S, Itoh Y, Yamaoka K, Toda H, Tanabe T (1996) Corticobasal degeneration: widespread argentophilic threads and glia in addition to neurofibrillary tangles. Similarities of cytoskeletal abnormalities in corticobasal degeneration and progressive supranuclear palsy. J Neurol Sci 138:66-77

110. Togo T, Sahara N, Yen SH, Cookson N, Ishizawa T, Hutton M, de Silva R, Lees A, Dickson DW (2002) Argyrophilic grain disease is a sporadic 4-repeat tauopathy. J Neuropathol Exp Neurol 61:547-556

111. Tolnay M, Sergeant N, Ghestem A, Chalbot S, De Vos RA, Jansen Steur EN, Probst A, Delacourte A (2002) Argyrophilic grain disease and Alzheimer's disease are distinguished by their different distribution of tau protein isoforms. Acta Neuropathol (Berl) 104:425-434

112. Tolnay M, Clavaguera F (2004) Argyrophilic grain disease: a late-onset dementia with distinctive features among tauopathies. Neuropathology 24:269-283

113. Uchihara T, Mitani K, Mori H, Kondo H, Yamada M, Ikeda K (1994) Abnormal cytoskeletal pathology peculiar to corticobasal degeneration is different from that of Alzheimer's disease or progressive supranuclear palsy. Acta Neuropathol (Berl) 88:379383

114. Uchihara T, Kondo H, Ikeda K, Kosaka K (1995) Alzheimer-type pathology in melanin-bleached sections of substantia nigra. J Neurol 242:485-489

115. Uchihara T, Nakamura A, Yamazaki M, Mori O (2000) Taupositive neurons in corticobasal degeneration and Alzheimer's disease-distinction by thiazin red and silver impregnations. Acta Neuropathol (Berl) 100:385-389

116. Uchihara T, Nakamura A, Yamazaki M, Mori O (2001) Evolution from pretangle neurons to neurofibrillary tangles monitored by thiazin red combined with Gallyas method and double immunofluorescence. Acta Neuropathol (Berl) 101:535-539

117. Uchihara T, Nakamura A, Yamazaki M, Mori O, Ikeda K, Tsuchiya K (2001) Different conformation of neuronal tau deposits distinguished by double immunofluorescence with AT8 and thiazin red combined with Gallyas method. Acta Neuropathol (Berl) 102:462-466

118. Uchihara T, Ikeda K, Tsuchiya K (2003) Pick body disease and Pick syndrome. Neuropathology 23:318-326

119. Uchihara T, Nakamura A, Mochizuki Y, Hayashi M, Orimo S, Isozaki E, Mizutani T (2005a) Silver stainings distinguish Lewy bodies and glial cytoplasmic inclusions: comparison between Gallyas-Braak and Campbell-Switzer methods. Acta Neuropathol (Berl) 110:255-260

120. Uchihara T, Shibuya K, Nakamura A, Yagishita S (2005b) Silver stains distinguish tau-positive structures in corticobasal degeneration/progressive supranuclear palsy and in Alzheimer's diseasecomparison between Gallyas and Campbell-Switzer methods. Acta Neuropathol (Berl) 109:299-305

121. Uchihara T, Tsuchiya K, Nakamura A, Akiyama H (2005c) Argyrophilic grains are not always argyrophilic-Distinction from neurofibrillary tangles of diffuse neurofibrillary tangles with calcification revealed by comparison between Gallyas and Campbell-Switzer methods. Acta Neuropathol (Berl) 110:158-164 
122. Uchihara T, Tsuchiya K, Nakamura A, Akiyama H (2005d) Silver staining profiles distinguish Pick bodies from neurofibrillary tangles of Alzheimer type: comparison between Gallyas and Campbell-Switzer methods. Acta Neuropathol (Berl) 109:483-489

123. Uchihara T, Nakayama H (2006) Familial tauopathy mimicking corticobasal degeneration an autopsy study on three siblings. J Neurol Sci 246:45-51

124. Vallet PG, Guntern R, Hof PR, Golaz J, Delacourte A, Robakis NK, Bouras C (1992) A comparative study of histological and immunohistochemical methods for neurofibrillary tangles and senile plaques in Alzheimer's disease. Acta Neuropathol (Berl) 83:170-178

125. Wilcock GK, Matthews SM, Moss T (1990) Comparison of three silver stains for demonstrating neurofibrillary tangles and neuritic plaques in brain tissue stored for long periods. Acta Neuropathol (Berl) 79:566-568

126. Williams DR, de Silva R, Paviour DC, Pittman A, Watt HC, Kilford L, Holton JL, Revesz T, Lees AJ (2005) Characteristics of two distinct clinical phenotypes in pathologically proven progressive supranuclear palsy: Richardson's syndrome and PSPparkinsonism. Brain 128:1247-1258
127. Wisniewski HM, Wen GY, Kim KS (1989) Comparison of four staining methods on the detection of neuritic plaques. Acta Neuropathol (Berl) 78:22-27

128. Yamaguchi H, Hirai S, Morimatsu M, Shoji M, Harigaya Y (1988) Diffuse type of senile plaques in the brains of Alzheimertype dementia. Acta Neuropathol (Berl) 77:113-119

129. Yamaguchi H, Hirai S, Shoji M, Harigaya Y, Okamoto Y, Nakazato Y (1989) Alzheimer type dementia: diffuse type of senile plaques demonstrated by beta protein immunostaining. Prog Clin Biol Res 317:467-474

130. Yamaguchi H, Haga C, Hirai S, Nakazato Y, Kosaka K (1990) Distinctive, rapid, and easy labeling of diffuse plaques in the Alzheimer brains by a new methenamine silver stain. Acta Neuropathol (Berl) 79:569-572

131. Yamamoto T, Hirano A (1986) A comparative study of modified Bielschowsky, Bodian and thioflavin S stains on Alzheimer's neurofibrillary tangles. Neuropathol Appl Neurobiol 12:3-9

132. Yoshida M (2006) Cellular tau pathology and immunohistochemical study of tau isoforms in sporadic tauopathies. Neuropathology 26:457-470 